\title{
Research and the Amenities of Railway Travel
}

$\mathrm{W}^{\mathrm{s}}$ live in an age of rapid change, due largely to the increasing readiness and rapidity with which the results of scientific progress and research are applied to the conditions of life ; and, in addressing the Institute of Transport on April 11, Sir Harold Hartley showed how the amenities of railway passen. ger travel are being improved. He confined his remarks to the aspect of the comfort of the passenger rather than to such desiderata as increased speed and safety. After indicating the several conditions which together determine the comfort of a railway journey at modern speeds, he proceeded to detail the steps being taken to ensure their maximum effectiveness.

Absence of vibration or irregular motion is probably the most important factor so far as the passenger's comfort is concerned, and although the difficulty of eliminating these increases greatly with increase of speed, much has been done in recent years to mitigate them. Smooth running depends on the design of rolling stock and railway track and on their maintenance, and was dealt with under three aspects: the motion of the wheel on the rail, the springing and coupling of the vehicles, and the effect of the permanent way. Over many years, a large number of experiments have been made with various designs of bogies and various conings of wheels in order to discover which type gives the best riding and the least wear, and standard practice has been based on the results.

More recently a complete theoretical and experimental investigation has been carried out in the Engineering Department of the University of Cam. bridge by Prof. C. E. Inglis and Dr. R. D. Davies, as a result of which the form of the sinuous path traversed by a pair of coned wheels and their axle has been determined. The results of their calculations were confirmed both in the laboratory and on the track and their theory suggests that, instead of coned wheels, cylindrical tyres should give complete immunity from lateral oscillations and 'bogie-hunting'. In agreement with this theory are the results of the cinematograph record taken by the Chicago, Milwaukee and North Shore Railway, which also showed that with the cylindrical wheel there was no regular oscillation and its flange rarely struck the rail. Experiments in the case of the L.M.S. train, Coronation Scot, with various conings and with cylindrical tyres proved the excellent riding qualities of the latter, and it was with these that the train was fitted when the record run from Euston to Crewe was made on November 16, 1936. Excessive flange wear, however, necessitated the trueing of the wheels after 20,000 miles service on the London-Glasgow run, and a compromise was then made of using a 1 in 100 coning, which gives almost as good riding at high speed without the disadvantage of excessive flange wear. Another method of avoiding oscillation is to mount the wheels so as to rotate independently. This eliminates 'bogie-hunting', there is no sliding motion on a curve and the riding of the coach is excellent, but the construction is much more complicated and the cost correspondingly high.

Coaches are also subject to oscillations due to impacts at rail joints and to other track irregularities. A combination of helical and laminated springs is used to damp out these; the former give elastic support while the latter damp out the vibrations. In addition, rubber is being used in seat and bed construction to eliminate vibration, the most effective material being rubber and metal or wood in alternate layers. Also associated with comfortable travel is the correct balance and centring of the coaches and the correct construction of the coupling and buffing gear holding the vehicles together and preventing surging and transverse oscillation. Accurate records of the riding qualities of different types of construction and of the deterioration due to wear are obtained by means of the Cambridge accelerometer which, by the movements of two weights held between stiff springs, prepares a graph of vertical and transverse oscillations and has thus helped greatly in effecting positive improvements.

The maintenance of the permanent way is an important factor in ensuring the comfort of the passenger. At high speeds, irregularities of the track lead to impacts which react so as to produce greater irregularity. Here, the Hallade recorder is used to give a continuous record on a strip of paper of the horizontal, vertical and rolling movements of the vehicle as it passes over the line and so shows where adjustment of the track is necessary. The Hallade method was used in improving the track for the accelerated timings of the Coronation Scot and, as a result, not only had the cant of rails to be readjusted to balance the centrifugal force but also the line had in places to be slewed to improve the alignment and transition curves had to be lengthened so that the train could take the curves smoothly and without rolling at high speed. On the rail joint problem, a mathematical and experimental research is being carried out at Cambridge, and its results are looked forward to with much interest, for each of these joints is a point of weakness in the track.

The reduction of noise is also receiving constant attention and is being effected, so far as possible, by eliminating its production, by absorbing it at its source, by avoiding its entry to the compartment, and by absorbing quickly such noise as does enter. Streamlining of coaches, welding of the track, the use of absorbent material for a top dressing on the track are some of the means in use. Heating and ventilation offer opportunities for the introduction of further amenities. The only satisfactory method of attaining steady conditions is by a closed system of air conditioning, but the weight and cost of the plant only justify its use in very hot and dry climates. Two proprietary systems of forced air ventilation are in use on main-line trains in Great.Britain. Indirect lighting is coming into use and the crack train of the Victorian Railways-the "Spirit of Progress"-has indirect lighting for general purposes and shoulder lights for reading.

Of the improvements made in the asthetic and general amenities the public is no doubt well aware. The more attractive external appearance of the trains, the materials and decorations used inside, the large windows, reduction of draughts, size and easy form of seats, all add to the enjoyment of the passenger, so that, in these unsettled hustling days, a long railway journey may be in some measure a comfortable relaxation and an escape from the disturbances of the world. 